\title{
Imprinted DNA methylation reconstituted at a non-imprinted locus
}

\author{
David H. Taylor ${ }^{1}$ (1), Chelsea M. McLean ${ }^{1,2}$, Warren L. Wu ${ }^{3}$, Alex B. Wang ${ }^{4}$ and Paul D. Soloway ${ }^{1,3,4^{*}}$
}

\begin{abstract}
Background: In mammals, tight regulation of cytosine methylation is required for embryonic development and celIular differentiation. The trans-acting DNA methyltransferases that catalyze this modification have been identified and characterized; however, these proteins lack sequence specificity, leaving the mechanism of targeting unknown. A cisacting regulator within the Rasgrf1 imprinting control region (ICR) is necessary for establishment and maintenance of local imprinted methylation. Here, we investigate whether 3-kb of sequence from the Rasgrfl ICR is sufficient to direct appropriate imprinted methylation and target gene expression patterns when ectopically inserted at the Wnt locus.

Results: The Rasgrf1 ICR at Wnt1 lacked somatic methylation when maternally transmitted and was fully methylated upon paternal transmission, consistent with its behavior at the Rasgrf1 locus. It was unmethylated in the female germline and was enriched for methylation in the male germline, though not to the levels seen at the endogenous Rasgrf1 allele. Wht1 expression was not imprinted by the ectopic ICR, likely due to additional sequences being required for this function.

Conclusions: We have identified sequences that are sufficient for partial establishment and full maintenance of the imprinted DNA methylation patterns. Because full somatic methylation can occur without full gametic methylation, we infer that somatic methylation of the Rasgrfl ICR is not simply a consequence of maintained gametic methylation.
\end{abstract}

Keywords: Imprinting control region, Rasgrf1, cis element, DNA methylation, CTCF, Wnt1

\section{Background}

Cytosine methylation $(5 \mathrm{mC})$ is vital for the regulation of development and other essential processes such as $\mathrm{X}$-chromosome inactivation, genomic imprinting, transposon silencing, and terminal differentiation. $5 \mathrm{mC}$ is usually associated with transcriptional repression, but is known to be a complex and dynamically regulated modification. Research into this regulation has included descriptive studies such as methylome mapping $[1,2]$ or functional studies of the proteins that regulate methylation in trans, such as DNMT1 [3], and isoforms of DNMT3 [4-6]. Few studies, however, have yielded much information about the cis-acting DNA sequences that target these trans-acting proteins to the DNA in a locus-, tissue-, or time-specific manner. These few informative

\footnotetext{
*Correspondence: soloway@cornell.edu; pds28@cornell.edu

1 Field of Genetics, Genomics, and Development, College of Agriculture and Life Sciences, Cornell University, Ithaca, NY, USA

Full list of author information is available at the end of the article
}

studies have used imprinted loci, which undergo highly regulated parental-specific, monoallelic methylation and expression. Cis-acting regulators of $5 \mathrm{mC}$ have been identified at Igf2r [7], Snrpn [8, 9], H19 [10-14], and Rasgrf1 [15-17], and in some cases, their mechanisms of action have been elaborated.

The Rasgrf1 ICR that lies 30-kb upstream of the paternally expressed Rasgrf1 gene has two components: a differentially methylated domain (DMD) and an adjacent series of 41-nt tandem repeats, $2 \mathrm{~kb}$ in length [15] (Fig. 1). The repetitive element, which is required for proper methylation establishment [15] and maintenance after fertilization [18], acts as a promoter for a piRNAtargeted noncoding RNA (pitRNA) that is transcribed across the DMD in e16.5 testes [16]. piRNAs normally silence transposable elements in the male germline; however, a subset of these primary piRNAs interact with two loci within the pitRNA, called sites 1 and 2. The pitRNA is subsequently processed to secondary piRNAs, and de 


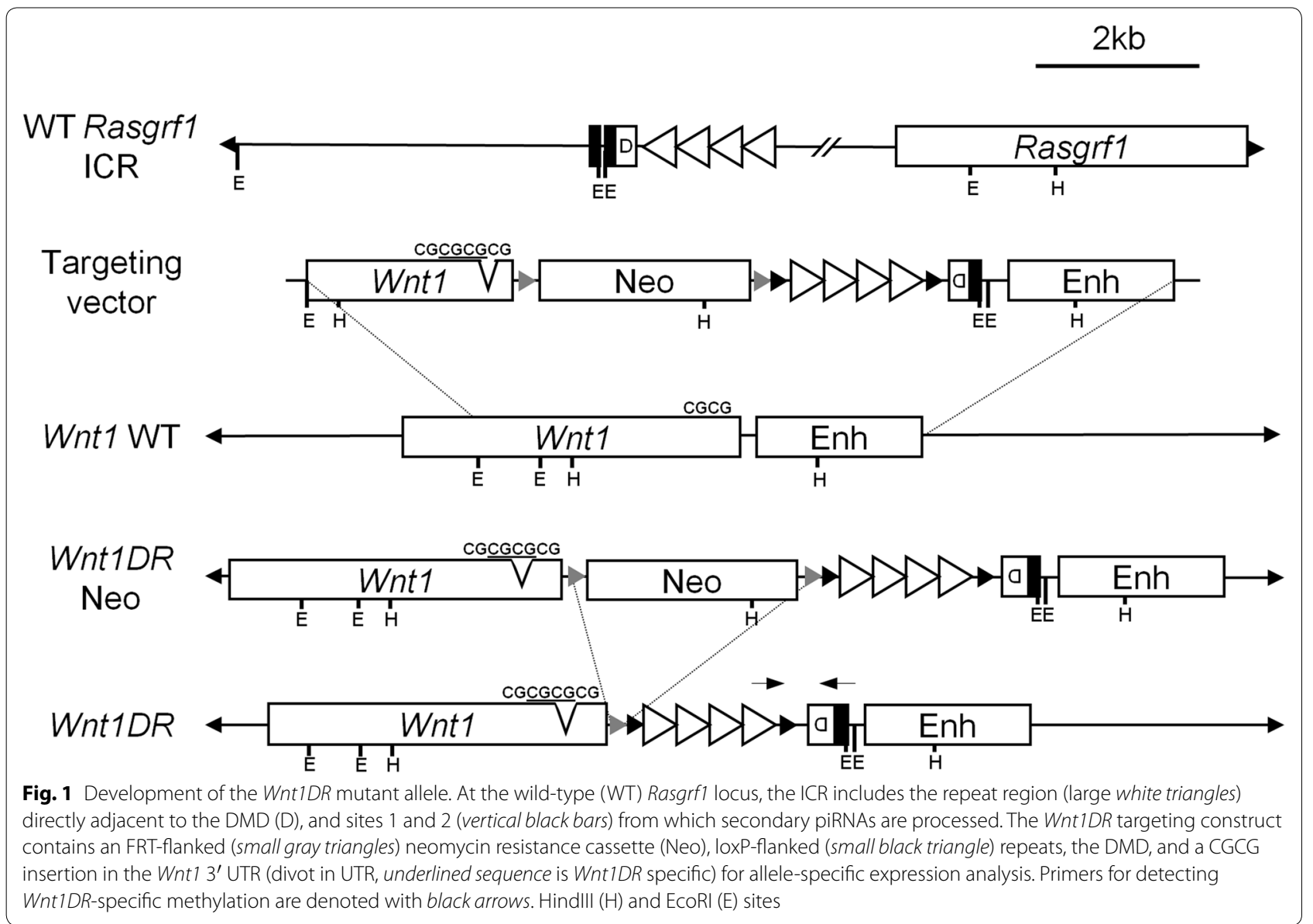

novo methylation of the ICR depends on piRNA pathway components MITOPLD and MILI.

Hypothesizing that repeat-mediated pitRNA transcription and the piRNA binding sites are sufficient to recapitulate imprinting, Park et al. [17] showed that proper sex-specific establishment of germline $5 \mathrm{mC}$ in transgenic reporter mice where the ICR was inserted between the $\gamma$-globin promoter and its enhancer. Proper somatic maintenance of $5 \mathrm{mC}$ was observed in subsequent generations if the construct was maternally transmitted; however, this construct was not sufficient to maintain an unmethylated maternal DMD in somatic tissue if it passed through the paternal germline. Failure of maintenance was likely due to the inappropriate methylation of a neomycin resistance selectable marker (neo) within the transgene which spread onto the adjacent ICR. In support of this, methylation analysis showed somatic methylation of the transgenic neo cassette upon maternal allele transmission, even if the transgenic DMD was unmethylated. These results confounded efforts to define the minimal sequences sufficient for Rasgrf1 imprinting.

In order to define the features of the Rasgrf1 ICR that are sufficient for imprinting control without confounding effects of sequences from other species or ambiguity of transgene insert sites, we targeted the ICR to the nonimprinted Wnt1 locus in mouse. Here, we show that site 1 and the repeats are sufficient to establish wild-type somatic imprinted methylation patterns at this ectopic locus. In keeping with previous studies, however, the imprinted expression patterns could not be recapitulated, possibly due to the complexity of the local chromatin context- or tissue-specific decreases in CTCF binding.

\section{Methods}

\section{Generation of targeted mice}

The $W n t 1^{\text {tmlpds }}$ vector was assembled using genomic clones from Rasgrf1, and PCR products from Wnt1. It included a loxP site at the Wnt1 DMD that enabled us to distinguish it from the endogenous Rasgrf1 DMD, and which we previously showed did not interfere with DNA methylation at Rasgrf1 [18]. The vector also included a 4-nt insertion at a MluI site in the Wnt1 3' UTR, creating a $B s s \mathrm{HII}$ site that enabled us to design allele-specific primers that distinguished transcripts from the endogenous and mutated Wnt1 locus. The vector was linearized with ZraI, electroporated into v6.5 ES cells, and placed 
under 200ug/ml G418 selection. Correct integration was verified by Southern blot after NdeI digestion using both a $5^{\prime}$ (amplified by PDS497 5'-GAAGTGGGGCACA TCATT and PDS492 5'-CATTTGCACTCTCGCACA) and $3^{\prime}$ (amplified by PDS349 5'-AATATGCCTGACGC ACCTTC and PDS 350 5'-CACTTCTCTCTGGGCC TCAC) external probes. The neo resistance cassette was removed using transient lipofection of the pCAGGSflpe-puro plasmid (Addgene \#20733). Cells were then microinjected into C2J blastocysts (Jax Stock No: 000058) and subsequently implanted into FVB pseudopregnant females. Germline transmission was verified by an internal PCR (PDS288 5'-TTACCCAGCTTCTCATAGGCGC and PDS1749 5'-CTGCAATTTCTGCCATCATC). Mice were then bred into the C57BL/6 background. The mutated Wnt 1 allele is referred to as Wnt1DR.

\section{Swim-up assay}

Swim-up assays were adapted from standard human protocols [19]. Briefly, sperm were isolated from cauda epididymis, washed in $1 \mathrm{ml}$ cell culture media supplemented with BSA, and pelleted gently at $300 \times g$ for $10 \mathrm{~min}$, after which the supernatant was discarded. After gently adding $300 \mathrm{ul}$ of fresh media, the pellet was incubated for $60 \mathrm{~min}$ at $30^{\circ} \mathrm{C}$ to allow motile sperm to enter the supernatant. The supernatant was then gently separated from the pellet, and both samples were processed for DNA methylation analysis.

\section{Methylation}

DNA for methylation analysis was bisulfite converted using the Zymo Methylation-Lightning kit (\#D5030) and amplified using allele-specific PCR for the Wnt1DR DMD (PDS405 5'-GTCGTTAAAGATAGTTTAGATA TGG and PDS2172-2175 5'-ACAACRAAATACRACAA TCACTAATAC) for 40 cycles. Oocyte DNA from 50 oocytes was pooled with salmon sperm as a carrier before conversion. Because of the exceedingly small amount of oocytes template, a nested approach was followed (PDS271 5'-GGAATTTTGGGGATTTTTTAGA GAGTTTATAAAGT and PDS2172-2175 5'-ACAACR AAATACRACAATCACTAATAC) for 15 additional cycles to improve yield. The bisulfite PCR products were purified (Qiagen PCR purification kit \#28104), end-polished (End-IT kit \#ER0720) for 45 min, A-tailed (NEB Klenow exo- \# M0212L) for $50 \mathrm{~min}$, and ligated with TruSeq adapters. This product was subjected to 10 rounds of amplification with barcode-specific primers (PDS2700 5'-AATGATACGGCGACCACCGA and PDS2701 5'-CAAGCAGAAGACGGCATACGA). Ampure beads (Agencourt \#A63880) were used to clean up between steps. The ligated product was then gel purified (Qiagen Gel extraction kit \# 28704) and quantified with the Qubit DNA HS kit (ThermoFisher \# Q32851) before pooling and sequencing using MiSeq. The resulting reads were quality controlled and trimmed, enabling the analysis of at least 1,000 high-quality sequences per sample (Summary Table of read results in Additional file 1) using a local installation of the Quantitative Methylation Analysis online software (QUMA http://quma.cdb.riken.jp/).

\section{Expression analysis}

cDNA was created via random hexamer reverse transcription from Trizol-extracted RNA derived from e9.5 mouse brains. Wnt1DR expression was measured by qRT-PCR and SYBR-green using primers specific to a four-nucleotide polymorphism in the $3^{\prime}$ UTR of the Wnt1DR allele (PDS2037 5'-CTGCCTCCTCA TCACTGTGTAAATA and PDS2039 5'-ATAACCGAA CGCGCGCGTG). Allele-specific expression was normalized to total Wnt1 expression (PDS2037 5'-CTG CCTCCTCATCACTGTGTAAATA and PDS20385'-CTG GAACCCAGCACAATAAATAGTTT).

\section{Chromatin immunoprecipitation (ChIP)}

CTCF ChIP was conducted using standard protocols. Briefly, brains from e9.5 mice carrying the $W n t 1 D R$ allele, transmitted either maternally or paternally, were finely minced smaller than $1 \mathrm{~mm}^{3}$, fixed for $10 \mathrm{~min}$ in a $1.1 \%$ formaldehyde solution, quenched with $2.5 \mathrm{M}$ glycine for $5 \mathrm{~min}$ at a final concentration of $125 \mathrm{mM}$, disaggregated using 30 strokes of a Dounce homogenizer, sonicated with the Covaris S2 Acoustic Disrupter (duty cycle $=5 \%$, intensity $=2$, cycles $/$ burst $=200$, cycle time $=3^{\prime}$ ON $/ 60^{\prime \prime}$ OFF, 3 cycles), and purified using $4 \mathrm{ul}$ of CTCF antibody [Kim et al. Cell. 128: 1231-45. (2007)] or IgG (Upstate \#12-371) on protein A beads (Life Technologies \#10003D) with multiple high stringency washes. Mouse embryonic fibroblasts (MEFs) were grown on 150 -mm plates ( 10 million/experiment) and subjected to the same ChIP protocol, except without a Dounce homogenization step. CTCF binding was verified using qPCR with primers specific to the H19 ICR as a positive control (PDS2825 5'-ATAGCCAAATCTGCACAG CG and PDS2826 5'-CATAAGGGTCATGGGGTGGT), an intergenic region as a negative control (from chr. 9 89646705-89646598, numbering based on NCBI37/ mm9; PDS2827 5'-AAGAAGCTGCTGAAACACCG and PDS2828 $5^{\prime}$-TGCTGGGTGGTACTGGTATG), and at the Wnt1DR DMD (PDS2846 5'-CGAAGTTATATCGATAA GCTGCTG and PDS2847 5'-CTACCGCTGCGCTAC AACTA).

\section{Results}

To test the sufficiency of the Rasgrf1 ICR to impart imprinting to an ectopic locus, we targeted the repeats and DMD to the Wnt1 locus. The Wnt1 gene was chosen 
for two reasons. First, the gene is haplosufficient, so monoallelic expression induced by imprinting is not lethal [20]. Second, the expression of the gene from e9.5 to e11.5 is completely dependent upon a well-defined enhancer $[21,22]$; therefore, the placement of methyl-sensitive enhancer-blocking sequences between the promoter and enhancer could plausibly control Wnt1 expression. The allele constructed, shown in Fig. 1, positioned the ICR $3^{\prime}$ of the Wnt 1 transcribed domain and $5^{\prime}$ of the enhancer, which lies $3^{\prime}$ of the gene body. The ICR was placed in the same orientation relative to the enhancer and promoter that it assumes at Rasgrf1. The vector was then linearized and electroporated into ES cells, which were subjected to G418 selection; and homologous recombination was verified by both Southern blot and PCR (Additional file 2). Because the neomycin resistance cassette, with its high GC enhancer and promoter, could alter regulation of the Wnt1 locus, we removed it using a FLPe expression vector prior to ES cell injections. Chimeras prepared by blastocyst injection transmitted the mutation through the germline. We refer to the allele as $W n t 1 D R(D R)$ indicating the presence of the DMD and the repeats at Wnt1.

Since pitRNA expression is necessary for proper DMD methylation in the male germline, we analyzed the e16.5 testis of heterozygous mutant animals. Allele-specific qRT-PCR after paternal transmission revealed that pitRNA expression from the Wnt1DR allele is less than $2 \%$ that of the endogenous allele within the same animals (Fig. 2). The Wnt1DR allele did not affect expression levels at the endogenous allele, as no significant difference could be found in wild-type pitRNA expression between animals that did or did not carry the allele.

We then set up pedigrees to fulfill three goals: first, to maintain the allele for six generations of passage through the female lineage; second, to maintain the allele for six generations of passage through the male lineage; and third, to alternate transmission through maternal and paternal lineages (Fig. 3c). Analysis of these pedigrees would reveal whether the Wnt1DR allele establishes and maintains the unmethylated state upon maternal transmission, the methylated state upon paternal transmission, and is properly reprogrammed upon each passage through the opposite sex's germline. To increase throughput for the methylation analysis, we developed a protocol for ligating TruSeq adapters onto the bisulfite PCR products for next-generation sequencing. Compared to the standard bisulfite analysis pipeline of cloning followed by Sanger sequencing, this new workflow yielded, on a per sample basis, vastly increased read counts (Additional file 1), decreased cost, and decreased hands-on effort.

To investigate establishment of DNA methylation at the $W n t 1 D R$ allele, we analyzed sperm and oocytes from mutant animals and observed significantly higher $5 \mathrm{mC}$

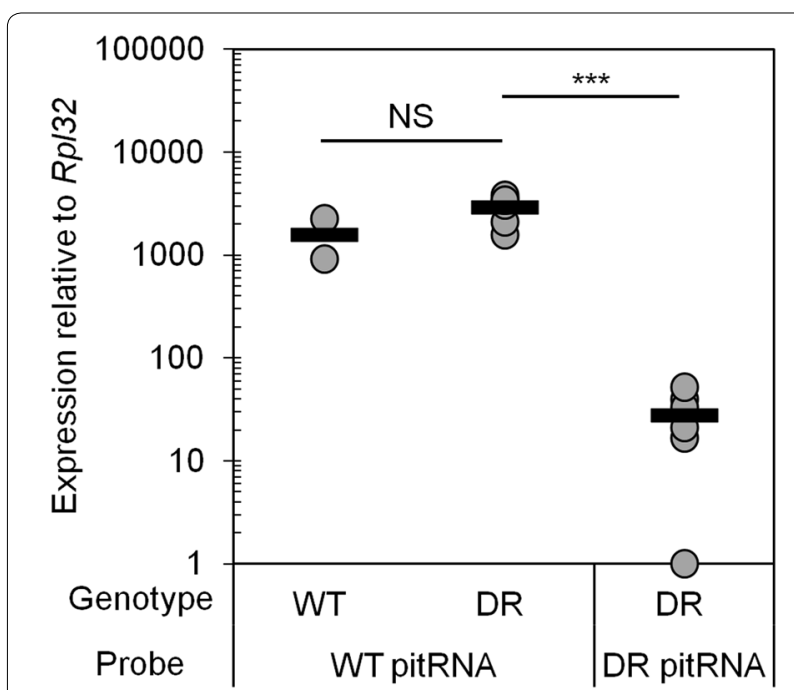

Fig. 2 pitRNA expression from the Wnt1DR allele is significantly lower than the endogenous allele. Allele-specific qRT-PCR for the Rasgrf1derived pitRNA (WT pitRNA) or the Wnt1DR-derived pitRNA (DR pitRNA) using $6+/ D R$ animals or 2 WT animals. Three asterisks represent $p<0.001$ using a two-tailed $t$ test, NS signifies not significant, and black horizontal lines indicate the average of relevant samples

in sperm compared to oocytes (Fig. 3a). Interestingly, at the Wnt1DR allele overall sperm methylation is $30 \%$, much lower than the $100 \%$ methylation levels seen at the endogenous Rasgrf1 locus. Methylation in sperm was not evenly distributed, with $41 \%$ of the reads containing no $5 \mathrm{mC}, 29 \%$ of the reads showing $66 \% 5 \mathrm{mC}$ or higher, and the remaining reads displaying intermediate levels (Fig. 3b and Additional file 2). We infer that incomplete establishment of methylation in sperm is due to significantly reduced expression of pitRNA (Fig. 2).

Analysis of methylation maintenance at the $W n t 1 D R$ mutant allele in adult tail DNA showed full recapitulation of both patterns of maternal and paternal inheritance seen at the wild-type Rasgrf1 ICR. The Wnt1DR allele preserved its unmethylated state in the soma during five generations of passage through the female germline (Fig. 3c). Transmission of the Wnt1DR allele through the male germline, despite low pitRNA expression and incompletely established germline methylation, displayed a fully methylated state in the soma throughout six generations of passage (Fig. 3c). Analysis of the alternating lineage pedigree demonstrated that the methylation profile could be faithfully reset at multiple different generations (Fig. 3c). These data demonstrate that the $\sim 3-\mathrm{kb}$ section of the ICR is sufficient for partial establishment, and full maintenance of the $5 \mathrm{mC}$ imprint. We extended these somatic DNA analyses, querying methylation states of DNAs from extra embryonic tissue, head, spine, and visceral organs taken from e9.5 embryos, the stage when the Wnt1 locus 


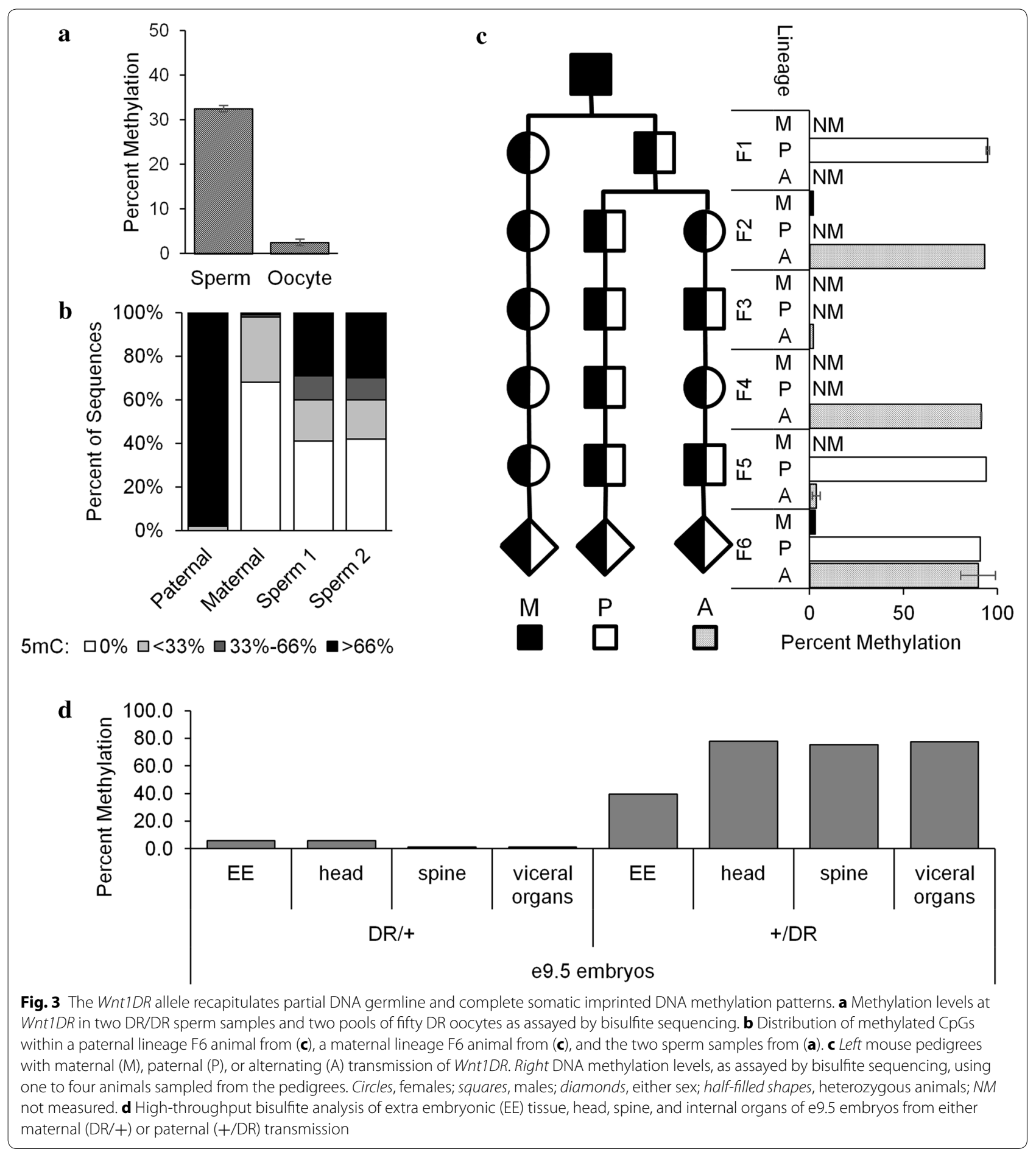

is expressed. We observed comparable patterns of methylation seen in adult tail DNAs: The Wnt1DR allele was significantly more methylated after paternal transmission than maternal transmission in each tissue (Fig. 3d). However, the levels of methylation in embryonic tissues were consistently lower than levels found in adult tail DNAs.
This is consistent with the possibility that in our system, methylation increases over developmental time. We did not assay methylation in preimplantation embryos and do not know whether the Wnt1DR allele resists global demethylation, as is typical for ICRs, or whether it undergoes demethylation along with the rest of the genome. 

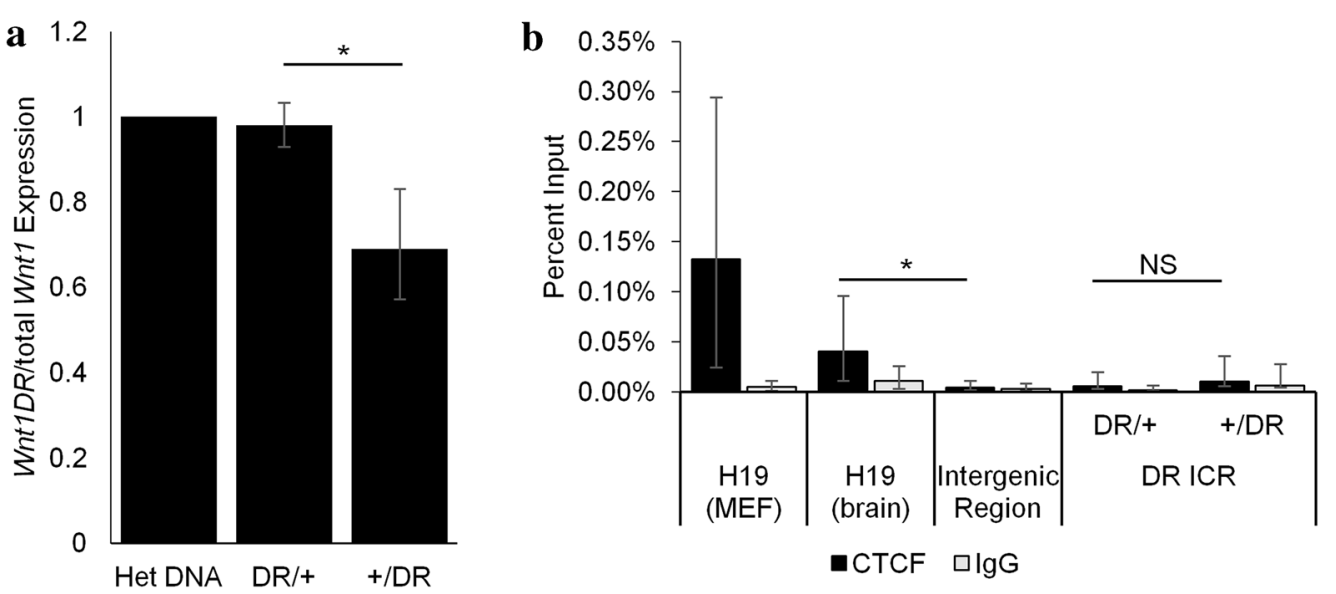

Fig. 4 Maternal transmission of Wnt1DR allele does not cause silencing or CTCF binding. a Allele-specific qRT-PCR using e9.5 brains taken from four mice with maternal (DR/+) or paternal (+/DR) transmission of Wnt1DR. Data are presented as the ratio of Wnt1DR allele-specific expression to total Wnt1 expression. Heterozygous DNA (Het DNA) was used as a calibrator for the 1:2 copy number of Wnt1DR to total Wnt1. Asterisk signifies $p<0.05$ using a two-tailed $t$ test. b CTCF ChIP. Chromatin from mouse embryonic fibroblasts (MEFs) or e9.5 brain (remaining samples) was subjected to ChIP using anti-CTCF or non-immune lgG. Purified DNAs were subjected to qPCR amplifying $\mathrm{H} 19$ as positive control for MEF and e9.5 brain chromatin samples, an intergenic region containing no CTCF sites as a negative control for, and the Wnt1DR allele. Three biological replicates were used. Asterisk represents $p<0.05$ using a two-tailed $t$ test after accounting for multiple hypothesis testing using Bonferroni's correction. NS not significant. Error bars denote standard deviation with error propagation

The abundance of unmethylated DNA in sperm, but its relative absence in adult somatic DNA after paternal transmission of the $W n t 1 D R$ allele (Fig. $2 \mathrm{~b}$ ), raised the possibility that unmethylated sperm may be less competent at fertilization and that adults analyzed were the result of fertilization by more competent and methylated sperm. To test this possibility, we isolated highly motile sperm from a sperm cell pellet by a swim-up assay and then compared the methylation state of the mobile sperm to that of sperm remaining in the cell pellet. Results showed that the mobile sperm were no more methylated than the sperm in the pellet (Additional file 3), indicating that unmethylated sperm were not at a motility disadvantage.

Somatic regulation of $5 \mathrm{mC}$ at the endogenous Rasgrf1 DMD is required to control the methylation-sensitive binding of CTCF. Unmethylated sequences permit CTCF binding and enhancer-blocking function to silence transcription from the maternal allele [23]. We therefore assayed expression of Wnt 1 from our construct to determine whether the faithfully imprinted $5 \mathrm{mC}$ at $W n t 1 D R$ enabled allele-specific expression. Our design of the Wnt1DR allele included placement of a four-nucleotide polymorphism in the $3^{\prime}$ UTR to facilitate allele-specific qRT-PCR analysis. We found that maternal transmission of the allele did not repress Wnt1 (Fig. 4a). Interestingly, we found that the paternal allele was slightly repressed, possibly due to the proximity of the Wnt1 gene and its enhancer to the highly methylated ICR.
There are two explanations for the inability to recapitulate imprinted expression. First, CTCF might not be bound to our mutant construct, possibly because the binding sites within the ICR are weak and might need external sequences for proper recruitment. CTCF has been shown to work in pairs to change the threedimensional structure of the DNA, so a second possibility might be that CTCF is correctly localized, but cannot function without interacting partner sites that can sequester the enhancer, as occurs at the globin locus control region [24]. To determine whether CTCF is bound to the unmethylated, maternally transmitted Wnt1DR allele, we performed ChIP-qPCR which revealed that while there was significant binding at the positive control (H19 ICR) in both mouse embryonic fibroblasts (MEFs) and e9.5 brains, there is no significant CTCF binding to the negative control or to the Wnt1DR ICR (Fig. 4b) in e9.5 brains. In MEFs, the endogenous Rasgrf1 ICR binds CTCF, whereas the ectopic Wnt1DR ICR does not (Additional file 4). Therefore, we conclude that the Wnt1DR allele either lacks CTCF-recruiting sequences that were present at the endogenous locus, that the novel chromosomal context at the Wnt1 locus acts to negatively regulate CTCF binding, or that overall binding of CTCF in e9.5 brain is sufficiently low that imprinted expression of $W n t 1$ is not possible, even if the required cis-acting elements for imprinting are present and functional. 


\section{Discussion}

Temporally controlled, tissue-specific targeting of DNA methylation to specific sequences is required for normal fertility and health in mammals. Very few systems have identified sequences which are sufficient to recruit epigenetic effectors of $5 \mathrm{mC}$, and even those sequences appear to act by a variety of mechanisms. Here, we investigate the Rasgrf1 imprinted region and define a 3-kb portion of the ICR that is sufficient to recapitulate many features of Rasgrf1 imprinting when exported to the ectopic Wnt1 locus.

In oocytes, only $2.5 \%$ of the CpGs in 19,418 bisulfite reads analyzed harbored $5 \mathrm{mC}$ at $W n t 1 D R$, consistent with the lack of $5 \mathrm{mC}$ in oocytes reported at Rasgrf1. Similarly, upon maternal transmission of Wnt1DR, only $2.6 \%$ of the CpGs in 65,497 bisulfite reads were methylated, also consistent with the methylation state at the maternal copy of Rasgrf1.

The pitRNA, whose expression in e16.5 testes is necessary in cis for imprinted DNA methylation at Rasgrf1 $[16,17]$, is expressed from Wnt1DR. However, its expression level is only $2 \%$ that seen from Rasgrf1. This weak expression may be due to undefined sequences at Rasgrf1 that are necessary for full expression, but are absent from the $W n t 1 D R$ allele. Alternatively, the necessary sequences may be present, but the chromatin context at Wnt1 may not be permissive for their full activity. A previously published allele [17] contained fewer sequences from the endogenous ICR relative to the Wnt1DR construct, but still established robust sperm methylation, arguing for the latter hypothesis. Despite the low level of pitRNA expression, it is possible that it was sufficient to establish methylation in the male germline at an average of $30 \%$ of the CpGs assayed at Wnt1DR, based on analysis of 6632 bisulfite sequencing reads from sperm DNA. Of those reads, $41 \%$ showed no $W n t 1 D R$ methylation, and an equal percentage showed methylation levels between 33 and $100 \%$. A similar pattern was revealed upon separation of sperm by motility, eliminating the possibility that sperm were unmethylated merely because of fundamental defects affecting their maturation to the motile stage. An alternative interpretation is that the pitRNA is dispensable for methylation at Wnt1.

Intriguingly, despite the abundance of unmethylated and partially methylated sperm, somatic methylation was nearly complete in 11 adult mice tested with a paternally transmitted Wnt1DR allele; $92 \%$ of CpGs assayed in 54,923 bisulfite reads were methylated. This result suggests either that the modest levels of pitRNA expression and the partial establishment of male germline methylation are sufficient for full somatic methylation, or that germline methylation is not necessary for full somatic methylation. In e9.5 embryonic tissues of mice with a paternally transmitted Wnt1DR allele, $75 \%$ of CpGs assayed in 22,162 bisulfite reads were methylated. When compared to the $92 \%$ rate in adult tissues, one might infer that acquisition of methylation is progressive after fertilization, rather than depending upon preexisting methylation in sperm. We cannot know both the methylation state of a given sperm, and the somatic methylation of the mouse resulting from its fertilization. It is possible that the 11 adult progeny assayed after paternal transmission of Wnt1DR arose only from the $59 \%$ of sperm that were richly methylated. However, assuming that fertilization fitness among sperm does not depend upon Wnt1DR methylation state, there is less than a $0.3 \%$ probability that this is the case. If germline methylation is necessary for somatic methylation, then in animals arising from fertilization by sperm lacking methylation on the Wnt1DR DMD, the somatic methylation may have occurred by methylation spreading from the repeats, sperm-transmitted pitRNA, or another unknown mechanism. Because of their repetitive nature, the repeats are difficult to characterize for methylation status; however, they are unlikely to exhibit a methylation profile which differs from the DMD because our assay spans the area between the repeats and pitRNA sites 1 and 2-the sequences which recruit DNA methylation. Sperm-transmitted RNAs are also unlikely, as piRNAs appear to be depleted in mature spermatozoa $[25,26]$. It is formally possible, however, that a limited number of piRNAs and their effector proteins are still active after fertilization.

Allele-specific histone modifications like H3K9me2 [27], H3R3me2 [28], H3K9me3 [29], or H4K20me2 [29], rather than $5 \mathrm{mC}$, could represent the primary imprinting mark, which subsequently enables somatic DNA methylation at Wnt1. Indeed, H3K9me2 is present on the Rasgrf1 ICR and has already been shown to be vital for imprinting maintenance; it recruits PGC7 which subsequently protects against Tet-mediated demethylation of the paternal allele after fertilization $[27,30]$. This mark could also be sufficient to recruit de novo DNA methylation to the paternal allele after fertilization in the absence of a preexisting methylation. Caution must be advised when interpreting histone immunoprecipitation experiments in sperm; however, as histones are switched for protamines in post-meiotic spermatids, leading to low total histone occupancy and inflated signal from rare epigenetic marks, compared to tissues lacking protamine substitutions. Analysis of the Rasgrf1 ICR in particular revealed a depletion of nucleosomes, potentially complicating any interpretation [31]. Our finding that somatic methylation of Wnt1DR may not depend on prior methylation establishment is 
consistent with reports of knock-in alleles of the $H 19$ ICR at $A f p$ [11] and CD3 [14], as well as other models $[12,13]$ which also displayed incomplete germline methylation followed by complete somatic methylation. It is also consistent with the work examining methylation acquisition of Igf $2 r$ ICR sequences, which was done by injecting DNA into post-fertilization embryos [7], not into oocytes where DNA methylation establishment normally occurs [32]. Finally, the possibility that somatic $5 \mathrm{mC}$ can arise in the absence of previously established $5 \mathrm{mC}$ in sperm is consistent with our previous findings that sperm methylation is not sufficient for somatic maintenance of that mark [18].

A previous transgenic model to test the sufficiency of the Rasgrf1 ICR for control of imprinted DNA methylation failed to exhibit reversible somatic methylation upon sequential passage through the male and female germlines [17]. Analysis of that model was confounded by persistent methylation of a neo reporter cassette. In contrast, the Wnt1DR allele exhibited the expected methylation reversal across six generations, demonstrating the sufficiency for somatic methylation imprinting of the tested sequences.

Although somatic methylation of the Wnt1DR allele faithfully recapitulated what was observed on the endogenous Rasgrf1 allele, this was not sufficient to impart imprinted expression to Wnt1. Imprinted expression at Rasgrf1 requires silencing of the maternal allele by CTCF binding to the DMD, which limits activity of the maternal promoter. Methylation at the paternal allele prevents CTCF binding, and enhancer-promoter interactions are unrestricted. The binding of CTCF to Wnt1DR was undetectable above background, which might account for the failure of the unmethylated maternal Wnt1DR allele to be silenced. Lack of CTCF binding is likely due to its complex role in mediating the formation of threedimensional chromatin structures with multiple interacting CTCF-binding sites [33-36]. The local chromosomal context at Wnt1 may lack appropriate partner sites for the Wnt1DR ICR and therefore discourage this complex binding. Alternatively, if CTCF-binding activity is limited in e9.5 brain, this might be sufficient to explain lack of imprinted expression at Wnt1DR.

\section{Conclusions}

We have identified sequences that are sufficient for partial germline, and full somatic $5 \mathrm{mC}$ imprinting at an ectopic locus. Rasgrf1 is one of the few loci whose cisacting sequences have been so narrowly defined. Discordance between the levels of Wnt1DR methylation in sperm and somatic tissue after paternal transmission is consistent with prior findings that post-fertilization methylation is not merely a maintenance of gametic methylation, indicating the existence of distinct post-fertilization methylation mechanisms.

\section{Additional files}

\begin{abstract}
Additional file 1. Breakdown of MiSeq methylation data. Libraries were analyzed in two separate runs. In the first run $\left(^{*}\right)$, all 6 libraries were placed into $<1 \%$ of a lane while in the second run $(\dagger)$, each library received $\sim 1 \%$ of a lane. Files from the second run were prohibitively large and were therefore truncated to $10 \mathrm{MB}$ ( $\sim 38,653$ sequences) before QUMA analysis. PHRED filter: $90 \%$ of the read $>20$. QUMA filter: $>98 \%$ identity, $<10$ mismatches, $>95 \%$ conversion, $<5$ unconverted $\mathrm{CpHs}$. Supernatant (sup.); animal from Figure 3C maternal lineage (M); animal from Figure 3C paternal lineage $(P)$; animal from Figure $3 C$ alternating lineage $(A)$.
\end{abstract}

Additional file 2. Confirmation of correct integration of Wht1DR construct. A) Schematic of Southern probes and internal PCR used in panels B and C. B) Southern blots on Ndel digested ES cell DNA were probed with either an external 5' probe or an external 3' probe with the expectation of a wild type $11.74 \mathrm{~kb}$ band and a mutant $9.76 \mathrm{~kb}$ and $7.47 \mathrm{~kb}$, respectively. Example of positives in lane 5 of both gels. C) Internal PCR as depicted in panel A was used to follow construct transmission. Rpl32 was used as a loading control.

Additional file 3. Distribution of methylation levels in sperm DNA. Rank order analysis (A) and bar charts (B) were used to give a more detailed view of the distribution of methylation levels within Sperm 1 and Sperm 2 samples described in Figure 3B. Sperm 3 and Sperm 4 are derived from swim up assays that separate motile (sup.) from non-motile (pellet) sperm.

Additional file 4. CTCF binding in MEF cells. CTCF ChIP was performed as in Figure 4, using MEF cells as a chromatin source. The endogenous Rasgrf1 ICR (WT ICR), shows significant CTCF binding; whereas the ectopic Wnt1DR ICR, shows no significant binding.

\begin{abstract}
Abbreviations
ICR: imprinting control region; $5 \mathrm{mC}$ : cytosine methylation; ADS: allelediscriminating sequence; DNS: de novo methylation signal; DMD: differentially methylated domain; pitRNA: piRNA-targeted RNA; neo: neomycin selection cassette; ChIP: chromatin immunoprecipitation; qRT-PCR: quantitative reverse transcription-polymerase chain reaction; $\mathrm{H} 3 \mathrm{~K} 9 \mathrm{me2}$ : histone three lysine nine dimethylation; H3R3me2: histone three arginine three dimethylation; $\mathrm{H} 3 \mathrm{~K} 9 \mathrm{me}$ : histone three lysine nine trimethylation; H4K20me2: histone four lysine twenty demethylation.
\end{abstract}

\section{Authors' contributions}

DHT, CM, and PDS designed experiments. DHT, CM, WLW, and ABW performed experiments. DHT and PDS wrote the manuscript. All authors read and approved the final manuscript.

\section{Author details \\ ${ }^{1}$ Field of Genetics, Genomics, and Development, College of Agriculture and Life Sciences, Cornell University, Ithaca, NY, USA. ${ }^{2}$ Present Address: Department of Molecular Oncology, The Netherlands Cancer Institute, Ples- manlaan, Amsterdam, The Netherlands. ${ }^{3}$ Division of Nutritional Sciences, Col- lege of Agriculture and Life Sciences, Cornell University, Ithaca, NY, USA. ${ }^{4}$ Field of Biochemistry, Molecular and Cell Biology, College of Agriculture and Life Sciences, Cornell University, Ithaca, NY, USA.}

\section{Acknowledgements}

We thank Erin Chu and Roman Spektor for their stimulating conversations that benefited both the science and aesthetics of this paper. We thank the Cornell Stem Cell and Transgenic Core Facility for making the Wnt1DR mice and the Cornell University Biotechnology Resource Center (BRC) for their assistance in high-throughput sequencing. Finally, we thank Victor Lobanenkov for the CTCF antibody.

\section{Competing interests}

The authors declare that they have no competing interests. 


\section{Availability of data and material}

The datasets supporting the conclusions of this article are available in the GEO repository [GSE79895 http://www.ncbi.nlm.nih.gov/geo/query/acc. cgi?acc=GSE79895]. Requests for materials should be directed to the corresponding author

\section{Ethical approval and consent to participate}

All animal studies were approved by the Institutional Animal Care and Use Committee protocol 2002-0075. No studies included human subjects.

\section{Funding}

Funding for this study was from the National Institutes of Health (RO1 GM10523 to PDS. DHT and CMM were supported by T32 HD057854; ABW was supported by T32 GM007273).

Received: 5 April 2016 Accepted: 14 September 2016

Published online: 22 September 2016

\section{References}

1. Lister R, Pelizzola M, Dowen RH, Hawkins RD, Hon G, Tonti-Filippini J, et al. Human DNA methylomes at base resolution show widespread epigenomic differences. Nature. 2009;462:315-22.

2. Xie W, Barr CL, Kim A, Yue F, Lee AY, Eubanks J, et al. Base-resolution analyses of sequence and parent-of-origin dependent DNA methylation in the mouse genome. Cell. 2012;148:816-31.

3. Howell CY, Bestor TH, Ding F, Latham KE, Mertineit C, Trasler JM, et al. Genomic imprinting disrupted by a maternal effect mutation in the Dnmt1 gene. Cell. 2001;104:829-38.

4. Bourc'his D, Xu GL, Lin CS, Bollman B, Bestor TH. Dnmt3L and the establishment of maternal genomic imprints. Science. 2001;22:22.

5. Hata K, Okano M, Lei H, Li E. Dnmt3L cooperates with the Dnmt3 family of de novo DNA methyltransferases to establish maternal imprints in mice. Development. 2002;129:1983-93.

6. Kaneda M, Okano M, Hata K, Sado T, Tsujimoto N, Li E, et al. Essential role for de novo DNA methyltransferase Dnmt3a in paternal and maternal imprinting. Nature. 2004;429:900-3.

7. Birger Y, Shemer R, Perk J, Razin A. The imprinting box of the mouse lgf2r gene. Nature. 1999:397:84-8.

8. Shemer R, Hershko AY, Perk J, Mostoslavsky R, Tsuberi B, Cedar H, et al. The imprinting box of the Prader-Willi/Angelman syndrome domain. Nat Genet. 2000;26:440-3.

9. Kantor B, Makedonski K, Green-Finberg Y, Shemer R, Razin A. Control elements within the PWS/AS imprinting box and their function in the imprinting process. Hum Mol Genet. 2004;13:751-62

10. Cranston MJ, Spinka TL, Elson DA, Bartolomei MS. Elucidation of the minimal sequence required to imprint $\mathrm{H} 19$ transgenes. Genomics. 2001;73:98-107.

11. Park KY, Sellars EA, Grinberg A, Huang SP, Pfeifer K. The H19 differentially methylated region marks the parental origin of a heterologous locus without gametic DNA methylation. Mol Cell Biol. 2004;24:3588-95.

12. Tanimoto K, Shimotsuma M, Matsuzaki H, Omori A, Bungert J, Engel JD, et al. Genomic imprinting recapitulated in the human \{beta\}-globin locus. Proc Natl Acad Sci USA. 2005:102:10250-5.

13. Matsuzaki H, Okamura E, Shimotsuma M, Fukamizu A, Tanimoto K. A randomly integrated transgenic $\mathrm{H} 19$ imprinting control region acquires methylation imprinting independently of its establishment in germ cells. Mol Cell Biol. 2009;29:4595-603.

14. Gebert C, Kunkel D, Grinberg A, Pfeifer K. H19 imprinting control region methylation requires an imprinted environment only in the male germ line. Mol Cell Biol. 2010;30:1108-15.

15. Yoon BJ, Herman H, Sikora A, Smith LT, Plass C, Soloway PD. Regulation of DNA methylation of Rasgrf1. Nat Genet. 2002;30:92-6.
16. Watanabe T, Tomizawa S, Mitsuya K, Totoki Y, Yamamoto Y, KuramochiMiyagawa S, et al. Role for piRNAs and noncoding RNA in de novo DNA methylation of the imprinted mouse Rasgrf1 locus. Science. 2011;332:848-52.

17. Park YJ, Herman H, Gao Y, Lindroth AM, Hu BY, Murphy PJ, et al. Sequences sufficient for programming imprinted germline DNA methylation defined. PLoS One. 2012;7:e33024

18. Holmes R, Chang Y, Soloway PD. Timing and sequence requirements defined for embryonic maintenance of imprinted DNA methylation at Rasgrf1. Mol Cell Biol. 2006;26:9564-70.

19. Jameel T. Sperm swim-up: a simple and effective technique of semen processing for intrauterine insemination. J Pak Med Assoc. 2008;58:71-4.

20. McMahon AP, Bradley A. The Wnt-1 (int-1) proto-oncogene is required for development of a large region of the mouse brain. Cell. 1990;62:1073-85.

21. Echelard Y, Vassileva G, McMahon AP. Cis-acting regulatory sequences governing Wnt-1 expression in the developing mouse CNS. Development. 1994;120:2213-24.

22. Danielian PS, Echelard Y, Vassileva G, McMahon AP. A 5.5-kb enhancer is both necessary and sufficient for regulation of Wnt-1 transcription in vivo. Dev Biol. 1997:192:300-9.

23. Yoon B-J, Herman H, Hu B, Park YJ, Lindroth AM, Bell A, et al. Rasgrf1 imprinting is regulated by a CTCF-dependent methylation-sensitive enhancer blocker. Mol Cell Biol. 2005;25:11184-90.

24. Splinter E, Heath H, Kooren J, Palstra RJ, Klous P, Grosveld F, et al. CTCF mediates long-range chromatin looping and local histone modification in the beta-globin locus. Genes Dev. 2006;20:2349-54.

25. Grivna ST, Beyret E, Wang Z, Lin H. A novel class of small RNAs in mouse spermatogenic cells. Genes Dev. 2006;20:1709-14.

26. Peng $H$, Shi J, Zhang $Y$, Zhang $H$, Liao $S$, Li W, et al. A novel class of tRNAderived small RNAs extremely enriched in mature mouse sperm. Cell Res. 2012;22:1609-12

27. Nakamura T, Liu YJ, Nakashima H, Umehara H, Inoue K, Matoba S, et al. PGC7 binds histone H3K9me2 to protect against conversion of $5 \mathrm{mC}$ to 5hmC in early embryos. Nature. 2012;486:415-9.

28. Girardot M, Hirasawa R, Kacem S, Fritsch L, Pontis J, Kota SK, et al. PRMT5mediated histone $\mathrm{H} 4$ arginine-3 symmetrical dimethylation marks chromatin at $\mathrm{G}+\mathrm{C}$-rich regions of the mouse genome. Nucleic Acids Res. 2014:42:235-48.

29. Delaval K, Govin J, Cerqueira F, Rousseaux S, Khochbin S, Feil R. Differential histone modifications mark mouse imprinting control regions during spermatogenesis. EMBO J. 2007;26:720-9.

30. Nakamura T, Arai $Y$, Umehara H, Masuhara M, Kimura T, Taniguchi $H$, et al. PGC7/Stella protects against DNA demethylation in early embryogenesis. Nat Cell Biol. 2007:9:64-71.

31. Erkek S, Hisano M, Liang CY, Gill M, Murr R, Dieker J, et al. Molecular determinants of nucleosome retention at $\mathrm{CpG}$-rich sequences in mouse spermatozoa. Nat Struct Mol Biol. 2013;20:868-75.

32. Stoger R, Kubicka P, Liu CG, Kafri T, Razin A, Cedar H, et al. Maternalspecific methylation of the imprinted mouse lgf2 $r$ locus identifies the expressed locus as carrying the imprinting signal. Cell. 1993;73:61-71.

33. Ling JQ, Li T, Hu JF, Vu TH, Chen HL, Qiu XW, et al. CTCF mediates interchromosomal colocalization between lgf2/H19 and Wsb1/Nf1. Science. 2006:312:269-72

34. Cuddapah S, Jothi R, Schones DE, Roh TY, Cui K, Zhao K. Global analysis of the insulator binding protein CTCF in chromatin barrier regions reveals demarcation of active and repressive domains. Genome Res. 2009:19:24-32

35. Handoko L, Xu H, Li G, Ngan CY, Chew E, Schnapp M, et al. CTCF-mediated functional chromatin interactome in pluripotent cells. Nat Genet. 2011:43:630-8

36. Gosalia N, Neems D, Kerschner JL, Kosak ST, Harris A. Architectural proteins CTCF and cohesin have distinct roles in modulating the higher order structure and expression of the CFTR locus. Nucleic Acids Res. 2014;42:9612-22. 